\title{
BMJ Open SARS-CoV-2 and influenza virus coinfection among patients with severe acute respiratory infection during the first wave of COVID-19 pandemic in Bangladesh: a hospital-based descriptive study
}

\author{
Zubair Akhtar (D) , ${ }^{1}$ Md Ariful Islam, ${ }^{1}$ Mohammad Abdul Aleem, ${ }^{1,2}$ \\ Syeda Mah-E-Muneer, ${ }^{1}$ M Kaousar Ahmmed, ${ }^{1}$ Probir K Ghosh, ${ }^{1}$ \\ Mustafizur Rahman, ${ }^{1}$ Mohammed Ziaur Rahman, ${ }^{1}$ Mariya Kibtiya Sumiya, ${ }^{1}$ \\ Md Mahfuzur Rahman, ${ }^{1}$ Tahmina Shirin, ${ }^{3}$ A S M Alamgir, ${ }^{3}$ Sayera Banu, ${ }^{1}$ \\ Mahmudur Rahman, ${ }^{1,4}$ Fahmida Chowdhury ${ }^{1}$
}

To cite: Akhtar Z, Islam MA, Aleem MA, et al. SARSCoV-2 and influenza virus coinfection among patients with severe acute respiratory infection during the first wave of COVID-19 pandemic in Bangladesh: a hospital-based descriptive study. BMJ Open 2021;11:e053768. doi:10.1136/ bmjopen-2021-053768

- Prepublication history and additional supplemental material for this paper are available online. To view these files, please visit the journal online (http://dx.doi.org/10.1136/ bmjopen-2021-053768)

Received 23 May 2021 Accepted 08 November 2021

Check for updates

(c) Author(s) (or their employer(s)) 2021. Re-use permitted under CC BY-NC. No commercial re-use. See rights and permissions. Published by BMJ.

For numbered affiliations see end of article.

Correspondence to

Dr Zubair Akhtar;

zakhtar@icddrb.org

\section{ABSTRACT}

Objective To estimate the proportion of SARS-CoV-2 and influenza virus coinfection among severe acute respiratory infection (SARI) cases-patients during the first wave of COVID-19 pandemic in Bangladesh.

Design Descriptive study.

Setting Nine tertiary level hospitals across Bangladesh. Participants Patients admitted as SARI (defined as cases with subjective or measured fever of $\geq 38 \mathrm{C}^{\circ}$ and cough with onset within the last 10 days and requiring hospital admission) case-patients.

Primary and secondary outcomes Proportion of SARSCoV-2 and influenza virus coinfection and proportion of mortality among SARI case-patients.

Results We enrolled 1986 SARI case-patients with a median age: 28 years (IQR: $1.2-53$ years), and $67.6 \%$ were male. Among them, 285 (14.3\%) were infected with SARSCoV-2; 175 (8.8\%) were infected with the influenza virus, and five $(0.3 \%)$ were coinfected with both viruses. There was a non-appearance of influenza during the usual peak season (May to July) in Bangladesh. SARS-CoV-2 infection was significantly more associated with diabetes $(14.0 \%$ vs $5.9 \%, p<0.001)$ and hypertension $(26.7 \%$ vs $11.5 \%$, $\mathrm{p}<0.001)$. But influenza among SARI case-patients was significantly less associated with diabetes $(4.0 \%$ vs $7.4 \%$, $\mathrm{p}=0.047)$ and hypertension $(5.7 \%$ vs $14.4 \%, \mathrm{p}=0.001)$. The proportion of in-hospital deaths among SARS-CoV-2 infected SARI case-patients were higher $(10.9 \%(n=31)$ vs $4.4 \%(n=75), p<0.001)$ than those without SARS-CoV-2 infection; the proportion of postdischarge deaths within 30 days was also higher $(9.1 \%(n=25)$ vs $4.6 \%(n=74)$, $\mathrm{p}=0.001$ ) among SARS-CoV-2 infected SARI case-patients than those without infection. No in-hospital mortality or postdischarge mortality was registered among the five coinfected SARI case-patients.

Conclusions Our findings suggest that coinfection with SARS-CoV-2 and influenza virus was not very common and had less disease severity considering mortality in

\section{Strengths and limitations of this study}

The study used data from a robust national surveillance system that follows the WHO recent recommended methodology and has been operational since 2007 as a part of the National Influenza Center of Bangladesh.

- The study adds baseline data about the prevalence, clinical features and mortality following SARS-CoV-2 and influenza virus coinfection among the general population during the first wave of the COVID-19 pandemic during 2020 in Bangladesh.

- SARS-CoV-2 and influenza virus detection tests were carried out in virology laboratory of International Centre for Diarrhoeal Disease Research, Bangladesh.

- The study did not screen and test for influenzalike illnesses in the outpatient departments whose illnesses were not severe enough to require hospitalisation.

- The virus detection estimates are based on only those who came to seek care in surveillance hospital sites and not those who did not seek care at all.

- The surveillance activities to enrol severe acute respiratory infection case-patients were conducted 6 days a week excluding the weekend.

Bangladesh. There was no circulating influenza virus during the influenza peak season during the COVID-19 pandemic in 2020. Future studies are warranted for further exploration.

\section{INTRODUCTION}

Seasonal influenza epidemics in temperate zones of the northern and southern hemispheres occur during their respective winters, 
from November to March in the northern hemisphere and April to September in the southern hemisphere. ${ }^{1-4}$ Bangladesh is a tropical country in the northern hemisphere, but the annual seasonal influenza epidemic occurs typically during the monsoon period, that is, from May to September, ${ }^{5}$ with influenza peak activity for 12.5 weeks on average spanning from May (epi-weeks 18) and July (epi-weeks 30.5 ) every year. ${ }^{6}$

Influenza season during 2019-2020 ended very early in China compared with previous years, ${ }^{7}$ and there was a sharp decline of influenza circulation in the USA and several Asian countries ${ }^{8-11}$ of the Northern Hemisphere, including in Bangladesh. ${ }^{6}$ Similar observations were also reported in the Southern Hemisphere countries of Australia, Chile, South Africa and New Zealand. ${ }^{12} 13$ This decline in influenza virus activity might be attributed to many factors, including the substantial outbreak of SARSCoV-2 as a global pandemic and public health efforts to control this virus. ${ }^{6} 81213$ Both influenza and SARS-CoV-2 produce similar clinical manifestations like-fever, cough, headache, muscle and joint pain, severe malaise, sore throat, runny nose, anosmia and ageusia. ${ }^{14}$ Furthermore, SARS-CoV-2 and influenza viruses share the common route of human-to-human transmission through aerosolised or respiratory droplets. ${ }^{15}$ Researchers have speculated about the possibilities of coinfection by both viruses since the beginning of the COVID-19 pandemic. ${ }^{14}$ Furthermore, there have been reports of coinfections during the early pandemic period. ${ }^{16}$ Yue et al reported a high rate of coinfection of SARS-CoV-2 and influenza viruses; $49.8 \%$ for influenza $A$ and $7.5 \%$ for influenza $B$ at the initial stage of pandemic. ${ }^{17}$

Influenza remains a major public health concern, and there is a high prevalence of comorbid conditions such as chronic respiratory or cardiovascular conditions and malnutrition, leading to an excess influenza burden ${ }^{18-20}$ in resource-poor settings like Bangladesh. Estimates of 2011-2012 data indicated seasonal influenza strains contributed to substantial mortality (6097 (95\% CI 2604 to 14 199) deaths in 2010-2011 and 16804 (95\% CI 8588 to 25019 ) deaths in 2011-2012) with $60 \%-80 \%$ of deaths among elderly aged $>60$ year. ${ }^{21}$ Together with a significant burden of Influenza in Bangladesh, on 8 March 2020, the first three laboratory-confirmed cases of SARS-CoV-2 were detected, and the SARS-CoV-2 has been circulating since then. ${ }^{22}$

WHO encouraged the detection of SARS-CoV-2, mainly using the Global Influenza Surveillance and Response System (GISRS) as the laboratories, sentinel sites and reporting platforms are the same for both influenza virus and SARS-CoV-2. ${ }^{23}$ In Bangladesh, hospital-based influenza surveillance (HBIS) has been operating as one of the components of the National Influenza Center (NIC), detecting both Influenza and SARS-CoV-2 and, among severe acute respiratory infection (SARI) case-patients and reporting to GISRS even during challenging situations of the COVID-19 pandemic. We explored this HBIS data based on SARI case-patients to describe rates, clinical

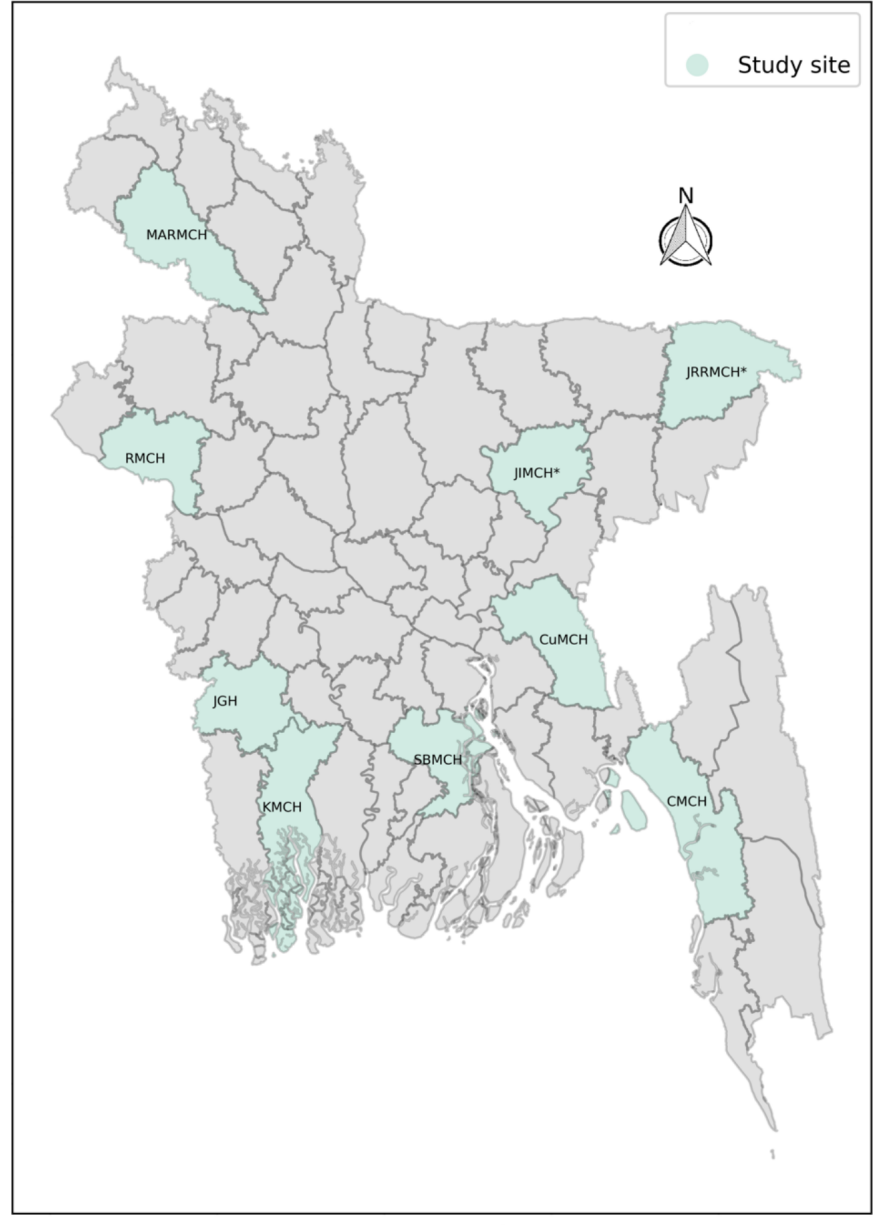

Figure 1 Study sites of the hospital-based influenza surveillance system: Public hospitals (1. RMCH: Rajshahi Medical College Hospital, Rajshahi; 2. CuMCH: Cumilla Medical College Hospital, Cumilla; 3. KMCH: Khulna Medical College Hospital, Khulna; 4. SBMCH: Sher-e-Bangla Medical College Hospital, Barishal; 5. CMCH: Chattogram Medical College Hospital, Chattogram; 6. MARMCH: M Abdur Rahim Medical College Hospital, Dinajpur; 7. JGH Jashore 250 bed General Hospital, Jashore) Private hospitals* (1. JIMCH: Jahurul Islam Medical College Hospital, Kishoregonj; 2. JRRMCH: Jalalabad Ragib-Rabeya Medical College Hospital, Sylhet.)

features and outcomes following SARS-CoV-2, influenza virus infections and coinfections by both viruses during the COVID-19 pandemic in 2020.

\section{METHODS}

\section{Surveillance sites and population}

The HBIS system in Bangladesh was initiated in 2007 as a part of NIC and during 2020 operated in nine sites (seven public and two private to have representation of case-patients from a socioeconomic perspective) tertiary level hospitals (figure 1) across Bangladesh through a collaboration between the Institute of Epidemiology, Disease Control and Research (IEDCR) of the Government of Bangladesh, the International Centre for Diarrhoeal Diseases Research, Bangladesh (icddr,b) and the 
US Centers for Disease Control and Prevention (US CDC). ${ }^{5}$ The surveillance remained operational 6 days a week (Saturday-Thursday) but is suspended during the weekend (Friday) and any national holidays. Detailed descriptions of surveillance systems have been described elsewhere. ${ }^{5}{ }^{24-26}$ Despite pandemic control efforts, the surveillance remained operational in inpatient departments of medicine and paediatrics wards, coronary care units, and specialised isolation wards established during the COVID-19 pandemic. This paper reports findings based on the patients enrolled during the COVID-19 pandemic between March and December 2020 only.

\section{Case identification}

Since the inception of the surveillance platform, 6 days a week during work hours (8:30-17:00 hours), study support staff and study physicians screen inpatients of medicine and the paediatric departments, coronary care units, and specialised isolation wards to identify casepatients with SARI, defined as subjective or measured fever of $\geq 38 \mathrm{C}^{\circ}$ and cough with onset within the last 10 days and requiring hospital admission. This case definition was adopted from $\mathrm{WHO}^{27}$ and used in this surveillance to screen participants since May 2016. During the COVID-19 pandemic from March 2020, study staff also screened for SARI case-patients in specialised isolation wards where suspected and probable COVID-19 patients were admitted.

\section{Specimen collection and laboratory analysis}

Study physicians, on written informed consent, collected nasopharyngeal (NP) and oropharyngeal (OP) swabs from all the enrolled SARI patients under all aseptic precautions using full personal protective equipment. Collected swabs were then stored in nitrogen dry shippers on-site and transported to icddr,b virology lab based in Dhaka every 2 weeks. Viral nucleic acid was extracted from $200 \mu \mathrm{L}$ of pooled NP and OP swab samples using InviMag Virus DNA/RNA Mini Kit (Invitek, STRATEC Molecular, Berlin-Buch, Germany) on Kingfisher Flex 96 (Thermo Fisher Scientific) automated nucleic acid extraction system according to the manufacturer's instructions. NP and OP swabs undergoing laboratory analysis were tested for seasonal influenza virus A, subtypes: A(H1N1)pdm09, A (H3N2), A(H5N1) and influenza virus $B$, lineages: Yamagata lineage and Victoria lineage by real-time reverse transcription-PCR (rRT-PCR) using primers and probes supplied by the US CDC. RNA was tested for SARS-CoV-2 by rRT-PCR targeting ORFlab-specific and N-gene-specific primers and probes following the protocol recommended by the Chinese Center for Disease Control and Prevention. Amplification was performed using the iTaq universal probes one-step Kit (Bio-Rad Laboratories, California, USA) in a Bio-Rad CFX96TM Real-Time PCR Detection System (Bio-Rad Laboratories).

\section{Data collection}

The surveillance physicians performed a physical examination of all enrolled SARI patients and collected data on a standardised surveillance report form on demographics, clinical presentations and diagnostics tests if available. Detailed information on demographic, clinical and diagnostic variables is described in our earlier published article describing HBIS. ${ }^{5}$ We also registered the status (full recovery, partial recovery, referral to another facility, and in-hospital death) of the enrolled participant at discharge and again (alive/deceased) after 30 days of discharge. Study staff made phone calls to patient/or family members to enquire about the patient's well-being after the SARI episodes. If a participant died within 30 days postdischarge, we registered the date, place of death and the family members' reported causes of death. Data collected were transferred in real time to our central server and have algorithms developed to report primary missing variables and/or values in variables that concern data quality (eg, age 115 years). Finally, during the later part of the workday, a data manager skimmed through the data, made a final check, followed up with the field team, and ensured correction of wrong entries to maintain a robust data management system.

\section{Data analysis}

We conducted descriptive analyses to describe the frequencies of SARI, influenza virus, influenza virus types and subtypes and SARS-CoV-2 virus infection. We have analysed through bivariate comparisons between proportion of laboratory-confirmed SARS-CoV-2 and Influenza infections and non-infected case-patients using Pearson's $\chi^{2}$ tests. For the time between illness onset and treatmentseeking variable, we performed the Mann-Whitney $\mathrm{U}$ non-parametric test to determine the differences between laboratory-confirmed influenza-positive and negative case-patients and also SARS-CoV-2-positive and negative case-patients. We plotted epidemiological curves describing the monthly SARS-CoV-2 and influenza virus circulation in Bangladesh. We also used Python V.3.6 to develop graphical illustration of study sites.

\section{Patient public involvement}

We developed research questions related to the influenza virus and SARS-CoV-2 detection according to the public health needs of Bangladesh. COVID-19 test results were immediately and confidentially shared with the Directorate General Health Services, Government of Bangladesh, they in term shared them with the individual patients. Anonymised influenza test results are regularly uploaded on the WHO's GISRS platform, on IEDCR and icddr,b websites.

\section{RESULTS}

\section{Demographic and clinical characteristics}

We identified a total of 2015 SARI case-patients; among the SARI case-patients, $11(0.5 \%)$ refused to provide 
Table 1 Demographic and clinical characteristics of SARI case-patients with SARS-CoV-2 and influenza virus infection in Bangladesh during March-December 2020

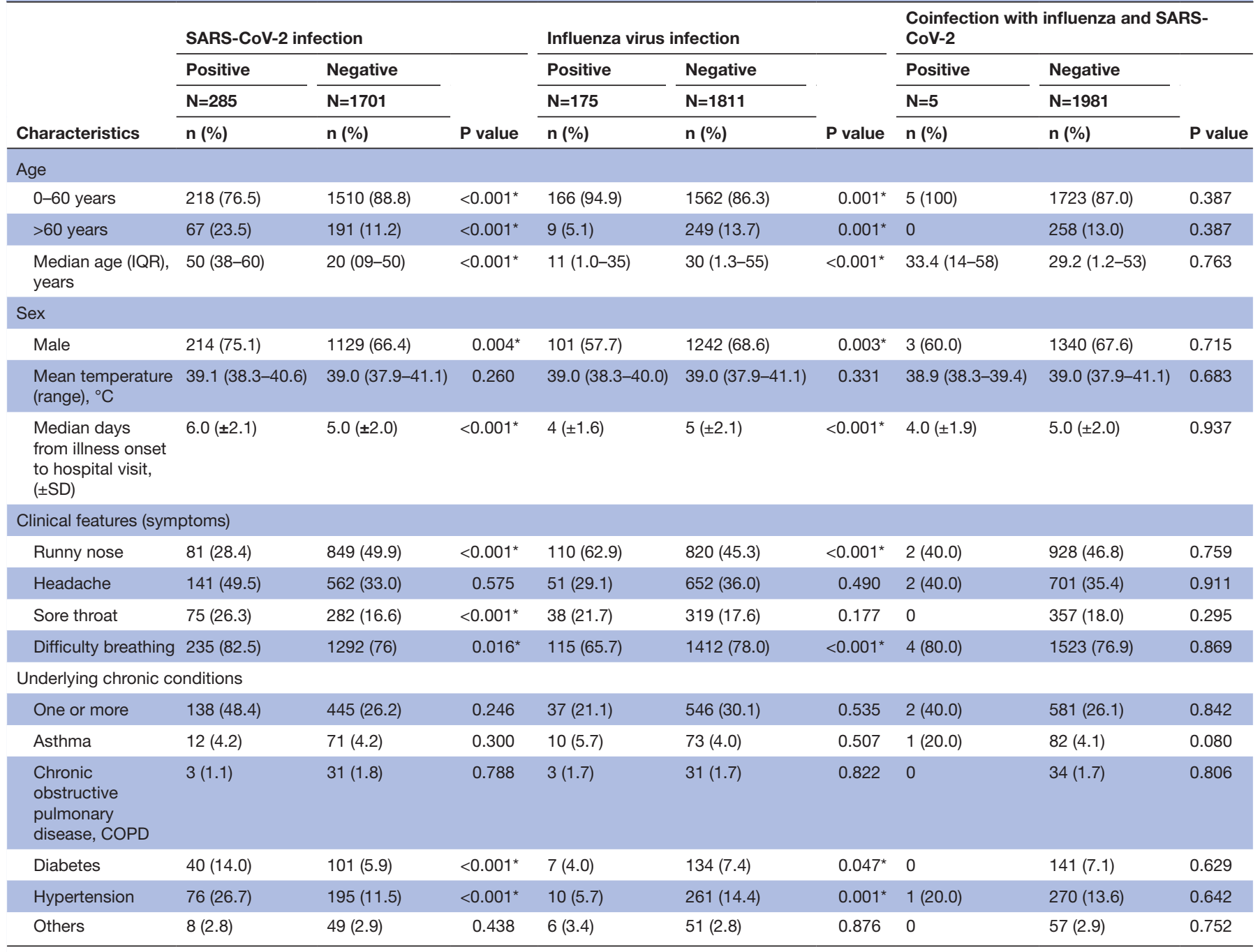

*Statistically significant $<0.05$.

consent, and $18(0.9 \%)$ were not enrolled due to other causes: absconded, discharged or referred to other facilities before sample collection. Finally, we enrolled in 1986 SARI case-patients with a median age of 28 years (IQR: 1.2-53); 67.6\% were males. Demographic and clinical information concerning the SARS-CoV-2 virus and influenza virus and coinfection are reported in table 1. There were 285 (14.3\%) SARI case-patients infected with SARS-CoV-2 and 175 (8.8\%) SARI case-patients infected with the influenza virus. Only five $(0.3 \%)$ SARI patients were coinfected with SARS-CoV-2 and influenza viruses. SARI patients of age group $>60$ were more likely to be infected with SARS-CoV-2 $(23.5 \% \quad(\mathrm{n}=67)$ vs $11.2 \%$ $(\mathrm{n}=191), \mathrm{p}<0.001))$ but less likely if age is $<60$ years $(76.5$ $(\mathrm{n}=218) \%$ vs $88.8 \% \quad(\mathrm{n}=1510), \mathrm{p}<0.001)$. Alternatively, those aged $>60$ years were more likely to be infected with the influenza virus than non-influenza case-patients (5.1\% $(\mathrm{n}=9)$ vs $13.7 \%(\mathrm{n}=249), \mathrm{p}<0.001))$. Males were more likely to be infected with the SARS-CoV-2 than SARS-CoV-2 non-infected case-patients $(75.1 \% \quad(\mathrm{n}=214)$ vs $66.4 \%$ ( $\mathrm{n}=1129), \mathrm{p}=0.004$ ) but less likely to be infected with the influenza virus compared with influenza negative SARI case-patients $(57.7 \%$ (101) vs $68.6 \%(1,242)$, $\mathrm{p}=0.003)$. Median duration $( \pm \mathrm{SD})$ from illness onset to hospital visit was longer $(6.0, \pm 2.1$ days vs $5.0, \pm 2.0$ days, $\mathrm{p}<0.001)$ for SARS-Cov-2-infected SARI patients but shorter $(4.0, \pm 1.6$ days vs $5.0, \pm 2.0$ days, $\mathrm{p}<0.001)$ for influenza virus-infected patients, compared with those without any SARS-CoV-2 and influenza virus infections, respectively. Fever and cough were present in all patients as we followed WHO SARI case definition; however, clinical symptoms were more likely to be present among SARSCoV-2-infected SARI patients compared with those without any SARS-CoV-2; difficulty breathing $(82.5 \% \quad(\mathrm{n}=235)$ vs $76.0 \%(\mathrm{n}=1292), \mathrm{p}=0.016)$ and sore throat $(26.3 \%(\mathrm{n}=75)$ vs $16.6 \%$ ( $\mathrm{n}=282), \mathrm{p}<0.001)$. Difficulty breathing was less likely among Influenza virus-infected SARI case-patients than those without $(65.7 \% \quad(\mathrm{n}=115)$ vs $78.0 \% \quad(\mathrm{n}=1412)$, $\mathrm{p}<0.001)$. Runny nose was more likely to be present among Influenza virus-infected SARI case-patients compared 


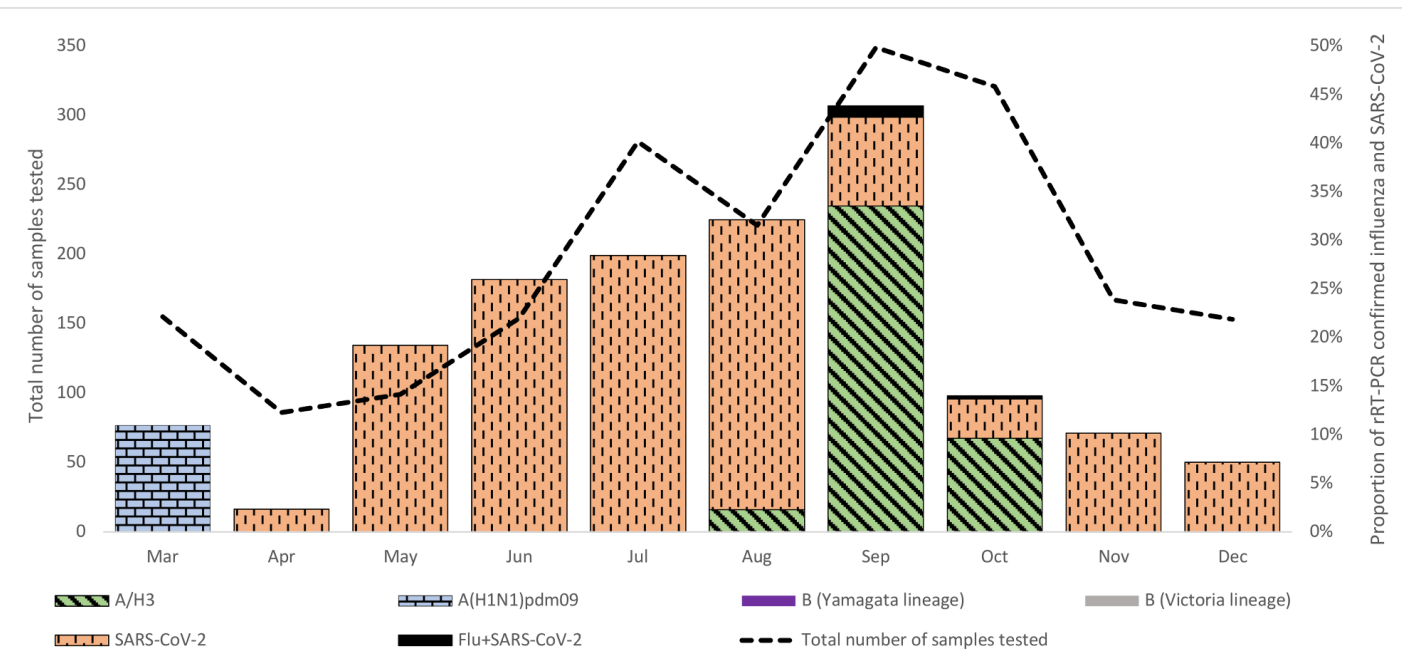

Figure 2 SARS-CoV-2, influenza virus and coinfection during March-December 2020 among SARI case patients in Bangladesh. SARI, severe acute respiratory infection.

with those without $(62.9 \% \quad(\mathrm{n}=110)$ vs $45.3 \% \quad(\mathrm{n}=820)$, $\mathrm{p}<0.001)$ but was less likely for SARS-CoV-2-infected ones than those without any SARS-CoV-2 $(28.4 \% \quad(\mathrm{n}=81)$ vs $49.9 \%(\mathrm{n}=849), \mathrm{p}<0.001)$. SARI case-patients with underlying chronic conditions were more likely to be infected with SARS-CoV-2 virus compared with those without any SARS-CoV-2 infection: diabetes $(14.0 \%(\mathrm{n}=40)$ vs $5.9 \%$ $(\mathrm{n}=101), \mathrm{p}<0.001)$ and hypertension $(26.7 \%(\mathrm{n}=76)$, vs $11.5 \%(\mathrm{n}=195), \mathrm{p}<0.001)$. In contrast, SARI case-patients with with underlying chronic conditions were less likely to be infected with influenza virus than without influenza virus infection: diabetes $(4.0 \%(\mathrm{n}=7)$, vs $7.4 \% \quad(\mathrm{n}=134)$, $\mathrm{p}=0.047)$ and hypertension $(5.7 \% \quad(\mathrm{n}=10)$, vs $14.4 \%$ $(\mathrm{n}=261), \mathrm{p}<0.001))$.

\section{Influenza virus and SARS-CoV-2 detection}

Only influenza $\mathrm{A}(\mathrm{H} 1 \mathrm{~N} 1)$ pdm09 was detected through the surveillance system in March 2020 (epi weeks 10-14). Then from epi week-15 onwards to epi week-32 (first week of April to the second week of August), no influenza virus was detected in the surveillance system in a range of 100-300 samples tested per month during the period. Later, only influenza A/H3N2 was detected again from epi week-33 onwards (mid-August), ranging from $2 \%$ to $34 \%$ proportion positive till epi week-44 (end of October 2020). Influenza/B lineages were not detected at all in Bangladesh during the COVID-19 pandemic in 2020 (figure 2). Six weeks after the first detection of SARSCoV-2 in Bangladesh, the first SARS-CoV-2 case-patient was detected in this surveillance system on epi week-17 (23 April 2020). According to different surveillance sites, the proportion of SARS-CoV-2 and influenza virus infection with coinfection of SARS-CoV-2 and influenza virus are reported in online supplemental table 1.

\section{Patient status at discharge and after $\mathbf{3 0}$ days postdischarge} There were 31 (10\%) in-hospital deaths registered among laboratory-confirmed SARS-CoV-2 case-patients, and in-hospital death was more likely among SARS-CoV-2 infected patients than non infected patients $(10.9 \%$ $(\mathrm{n}=31)$ vs $4.4 \%(\mathrm{n}=75), \mathrm{p}<0.001)$. However, for Influenza virus-infected SARI case-patients, in-hospital death was lower than Influenza negative patients $(1.1 \% \quad(\mathrm{n}=2)$ vs $5.7 \%(\mathrm{n}=104), \mathrm{p}=0.010)$. SARS-CoV-2 and influenza virusinfected case-patients were more likely to be referred to other healthcare facilities than SARS-CoV-2 and Influenza virus-negative case-patients, respectively $(33.3 \% \quad(\mathrm{n}=95)$ vs $3.4 \%(\mathrm{n}=58), \mathrm{p}<0.001 ; 2.9 \% \quad(\mathrm{n}=5)$ vs $8.2 \% \quad(\mathrm{n}=148)$, $\mathrm{p}=0.012$, respectively). At 1-month postdischarge there were $114(5.7 \%)$ case-patients lost to follow-up. We registered another 25 deaths $(9.1 \%)$ in SARS-CoV-2-infected case-patients, which was significantly higher compared with SARI case-patients without SARS-CoV-2 infection $(9.1 \%(\mathrm{n}=25)$ vs $4.6 \%(\mathrm{n}=74),<0.001)$. No in-hospital mortality or postdischarge mortality was registered among the five SARS-CoV-2 and Influenza virus coinfected SARI cases-patients (table 2).

\section{DISCUSSION}

We found a very low proportion of SARS-CoV-2 and influenza virus coinfection among SARI case-patients. Early studies reported a similarly low proportion of coinfection with influenza virus in the USA $(0.9 \%)$ and China $(0.4 \%) .{ }^{162829}$ However, later studies from larger cohorts reported a higher proportion $(52 \%)$ of influenza virus infection among laboratory-confirmed SARS-CoV-2 infected cases and $20 \%$ coinfection with other respiratory pathogens in China and the USA, respectively. They did not report the clinical prognosis among the coinfected cases. ${ }^{28}{ }^{30}$ Following the clinical course of SARI patients was beyond the scope of our study. Furthermore, with only the five cases of SARS-CoV-2 and influenza virus coinfection, our findings were inadequate to draw inference whether coinfected cases had a worse off clinical 
Table 2 Discharge and 1-month postdischarge outcomes among SARI case-patients with SARS-CoV-2 and influenza virus infection during March-December 2020 in Bangladesh

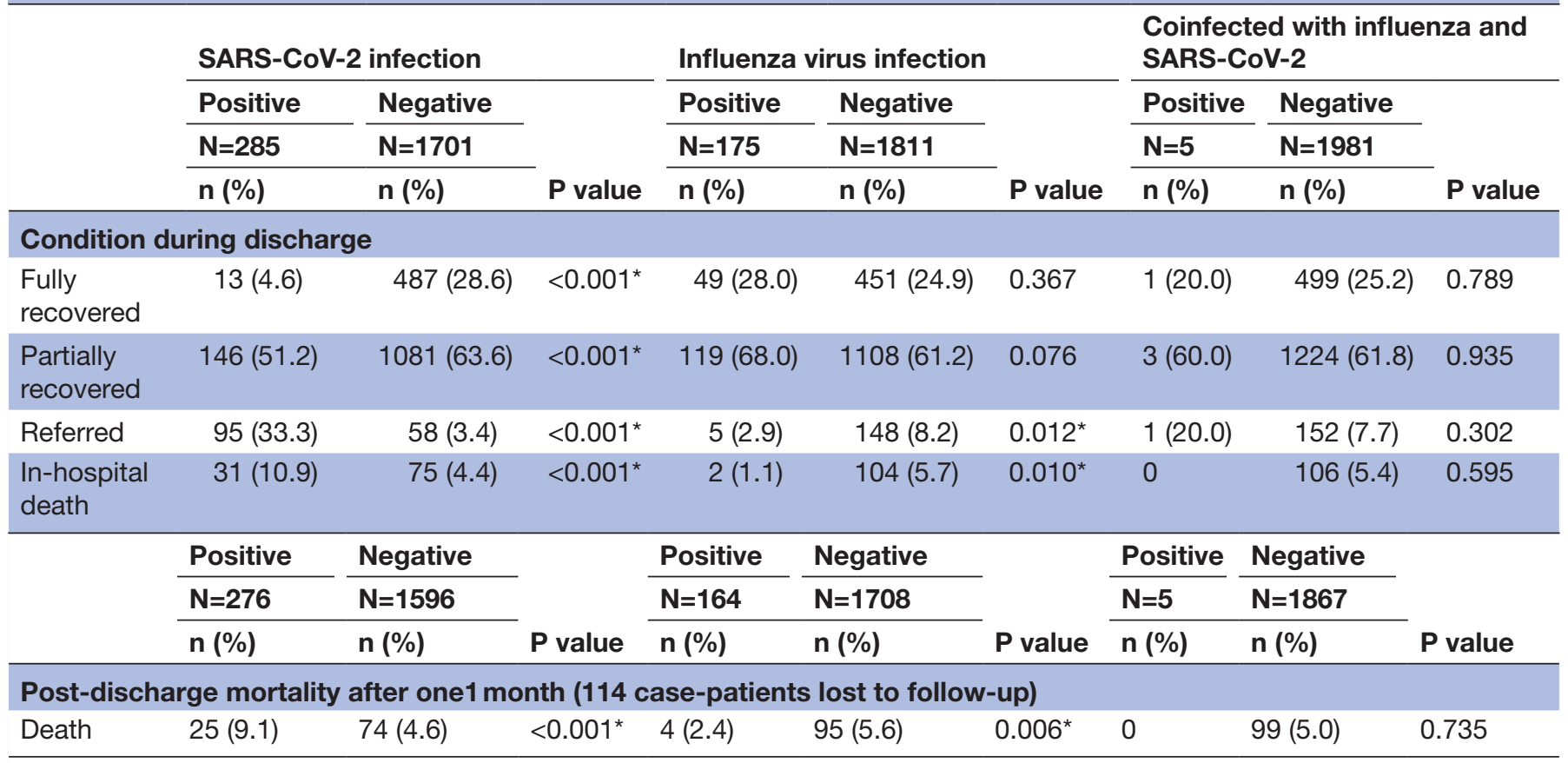

*Statistically significant $<0.05$.

SARI, severe acute respiratory infection.

prognosis or not. Future studies on larger cohorts and the clinical prognosis of coinfected patients are warranted.

During the 12.5 weeks (May-July) of peak influenza circulation time in Bangladesh, ${ }^{6}$ we did not detect any circulating influenza till 14 weeks from the start of the peak influenza season. Several public health control efforts in Bangladesh were undertaken from the mid-March epi (week-13) to control the COVID-19 pandemic. ${ }^{31}$ Notable ones included: closure of educational institutes, suspension of any political, religious, social and cultural gathering including state public programme and events, closure of transport services including domestic and international flights, and finally closure of all public and private offices except for hospitals, kitchen markets, drug stores and emergency services. A general holiday was issued from March 26 and extended several times till May 30 with mass communications regarding social distancing, wearing face masks and frequent hand washing messages. ${ }^{31}$ With the emergence of the SARS-CoV-2 virus in Bangladesh in the first week of March 2020, the influenza virus seemed to have disappeared and was not detected from the first week of April. The first reported case of influenza was from another study in Bangladesh on epi-week 29 of $2020,{ }^{32}$ which was detected 4 weeks ahead of this surveillance platform. In Bangladesh, there is no routine vaccination for influenza. ${ }^{33}$ Since both SARS-CoV-2 and influenza viruses have similar modes of transmission through respiratory and contact routes, ${ }^{14}$ these public health pandemic control efforts may have completely limited the transmission of influenza virus circulation in Bangladesh during its peak season. ${ }^{6}$ Similar marked influenza circulation reductions were also reported in Singapore, Thailand, China, Taiwan and New Zealand during the COVID-19 pandemic citing as a collateral effect of pandemic control measures as the predominant cause..$^{8-11} 13$

Since we enrolled all SARI case-patients, fever and cough were common in all SARS-CoV-2 infected patients, with difficulty breathing and sore throat as significant clinical features. During the early pandemic period, Chinese researchers reported fever and cough to be the most prevalent symptoms. ${ }^{35}$ Also, a meta-analysis during the early pandemic period reported difficulty breathing $(46 \%)$ as the most pervasive symptom after fever $(89 \%)$ and cough $(58 \%) \cdot{ }^{36}$ Our findings were consistent with these reported findings. Regarding demographics, the elderly aged $>60$ years were more likely to get infected with SARS-CoV-2 infection. In early reports from China, older age has been considered a significant risk factor for COVID-19 disease. ${ }^{37}$ In our study, males were also more likely to be infected. Our findings are consistent with findings from Italy ${ }^{38}$ and also from national-level data of Bangladesh, ${ }^{22}$ where more males being infected were reported than females. We found diabetes and hypertension as significant comorbid conditions among SARS-CoV-2 infected patients. Comorbid conditions with diabetes and hypertension are the most frequently reported comorbidities for COVID-19 in reports from China $^{37} 39$ and the USA. ${ }^{40}$ Therefore, it is highly recommended that older people with diabetes and hypertension take necessary precautions to protect themselves against SARS-CoV-2 infection. ${ }^{41}$ 
Our in-hospital mortality proportion was much lower than in other parts of the world during the start of the pandemic. Early retrospective studies among three cohorts from China reported 12\%, 17\% and $28 \%$ in-hospital mortality, ${ }^{41-43}$ while another study from the USA reported $24 \%$ in-hospital mortality among laboratoryconfirmed SARS-CoV-2 infection cases. ${ }^{42}$ We could not find any literature comparing our 30 days' postdischarge mortality, which was $9.1 \%$ of the laboratory-confirmed SARS-CoV-2 infection cases. Considering the low number of deaths (in hospital $n=31$ and postdischarge $n=25$ ), we could not identify any demographic or clinical characteristics that are likely to play a significant role in determining mortality both in-hospital or postdischarge. More extensive formative studies are recommended to better understand context-specific factors that may influence survival status following SARS-CoV-2 infection in Bangladesh.

Limitations to acknowledge first were not screening and testing for influenza-like illnesses in the outpatient departments whose illness was not severe enough to require hospitalisation. Second, using a specific WHO case definition of SARI probably made it more likely to miss actual COVID-19 case-patients, which might have had either fever or cough as the only presenting symptom. Third, the virus detection estimates are based on only those who came to seek care in surveillance hospital sites and not those who did not seek care at all. Lastly, six workdays a week, and this surveillance platform was subjected to under-reporting of cases. ${ }^{43}$ All the above may be accountable for having minimal estimates of influenza/ SARS-CoV-2 detections. Our study did not document the treatment provided, health-seeking behaviours and access to healthcare of the study participants, which may have confounded the estimates of in-hospital deaths and postdischarge mortality. Furthermore, associating these deaths to SARS-CoV-2/influenza virus infection alone was impossible as verbal autopsies were not performed.

In conclusion, our findings suggest that coinfection with SARS-CoV-2 and influenza virus was not very common in Bangladesh and had less disease severity considering mortality. There was an apparent absence of influenza virus circulation during the peak influenza season in Bangladesh. Studies exploring possible causes of the marked decrease of influenza virus infections during the COVID-19 pandemic are warranted to better understand the association of the COVID-19 pandemic and reduction of influenza virus infection.

\section{Author affiliations}

${ }^{1}$ Infectious Diseases Division, International Centre for Diarrhoeal Disease Research, Bangladesh (icddr,b), Dhaka, Bangladesh

${ }^{2}$ School of Population Health, University of New South Wales (UNSW), Sydney, Bangladesh

${ }^{3}$ Institute of Epidemiology, Disease Control and Research (IEDCR), Dhaka,

Bangladesh

${ }^{4}$ Global Health Development, EMPHNET, Dhaka, Bangladesh

Acknowledgements The authors are grateful to the study data collection team and study participants for their valuable data. We also acknowledge the support of participating tertiary-level hospital authorities and physicians involved in conducting this study

Contributors ZA was the lead in implementing this study, wrote the paper and performed data analysis. The protocol was drafted by FC, ZA, MAI, SM-E-M, MuR, MZR and MaR. MAI, MAA and SM-E-M also contributed to implementing the study and writing this paper. MKA contributed to project implementation and managed data. PKG developed data visualisation and was responsible for statistical analyses. MuR, MZR, MKS and MMR performed laboratory analysis. MAA, TS, ASMA, SB and $M a R$ provided critical feedback in implementing and writing this paper. FC was the principal investigator $(\mathrm{PI})$ of the study, provided overall guidance in implementing this study and in writing this paper. FC is the guarantor.

Funding This hospital-based influenza surveillance was funded by the Influenza Division of the Centers for Disease Control and Prevention (CDC), Atlanta, under the co-operative agreement (6 NU51IP000852-05-01). icddr,b is grateful to CDC for this support. icddr,b is also grateful to the Governments of Bangladesh, Canada, Sweden and the UK for providing core/unrestricted support.

Map disclaimer The depiction of boundaries on the map(s) in this article does not imply the expression of any opinion whatsoever on the part of BMJ (or any member of its group) concerning the legal status of any country, territory, jurisdiction or area or of its authorities. The map(s) are provided without any warranty of any kind, either express or implied.

Competing interests None declared.

Patient consent for publication Consent obtained directly from patient(s)

Ethics approval The study was approved by the icddr,b institutional review board prior to enrolling participants; US CDC's Human Research Protection Office approved a continuing reliance on the icddr,b IRB. Informed written consent to participate in the study was obtained.

Provenance and peer review Not commissioned; externally peer reviewed.

Data availability statement Data are available on reasonable request. Data generated during the study are subject to a data access policy of icddr,b and are available from icddrb's research administration on reasonable request through the corresponding author.

Supplemental material This content has been supplied by the author(s). It has not been vetted by BMJ Publishing Group Limited (BMJ) and may not have been peer-reviewed. Any opinions or recommendations discussed are solely those of the author(s) and are not endorsed by BMJ. BMJ disclaims all liability and responsibility arising from any reliance placed on the content. Where the content includes any translated material, BMJ does not warrant the accuracy and reliability of the translations (including but not limited to local regulations, clinical guidelines, terminology, drug names and drug dosages), and is not responsible for any error and/or omissions arising from translation and adaptation or otherwise.

Open access This is an open access article distributed in accordance with the Creative Commons Attribution Non Commercial (CC BY-NC 4.0) license, which permits others to distribute, remix, adapt, build upon this work non-commercially, and license their derivative works on different terms, provided the original work is properly cited, appropriate credit is given, any changes made indicated, and the use is non-commercial. See: http://creativecommons.org/licenses/by-nc/4.0/.

Author note $\mathrm{FC}$ was the principal investigator (PI) of the study.

ORCID iD

Zubair Akhtar http://orcid.org/0000-0001-5054-9243

\section{REFERENCES}

1 Hampson AW. Vaccines for pandemic influenza. The history of our current vaccines, their limitations and the requirements to deal with a pandemic threat. Ann Acad Med Singap 2008;37:510.

2 Tamerius J, Nelson MI, Zhou SZ, et al. Global influenza seasonality: reconciling patterns across temperate and tropical regions. Environ Health Perspect 2011;119:439-45.

3 Azziz Baumgartner E, Dao CN, Nasreen S, et al. Seasonality, timing, and climate drivers of influenza activity worldwide. J Infect Dis 2012;206:838-46.

4 Saha S, Chadha M, Al Mamun A, et al. Influenza seasonality and vaccination timing in tropical and subtropical areas of southern and south-eastern Asia. Bull World Health Organ 2014;92:318-30.

5 Zaman RU, Alamgir ASM, Rahman M, et al. Influenza in outpatient ILI case-patients in national hospital-based surveillance, Bangladesh, 2007-2008. PLoS One 2009;4:e8452. 
6 Akhtar Z, Chowdhury F, Rahman M, et al. Seasonal influenza during the COVID-19 pandemic in Bangladesh. PLoS One 2021;16:e0255646.

7 Liu M, Deng L, Wang D, et al. Influenza activity during the outbreak of coronavirus disease 2019 in Chinese mainland. Biosaf Health 2020;2:206-9.

8 Soo RJJ, Chiew CJ, Ma S. Decreased influenza incidence under COVID-19 control measures, Singapore. Emerg Infect Dis 20201933;26:1933-5.

9 Suntronwong N, Thongpan I, Chuchaona W, et al. Impact of COVID-19 public health interventions on influenza incidence in Thailand. Pathog Glob Health 2020;114:225-7.

10 Lei $\mathrm{H}$, Xu M, Wang X, et al. Nonpharmaceutical interventions used to control COVID-19 reduced seasonal influenza transmission in China. J Infect Dis 2020;222:1780-3.

11 Kuo S-C, Shih S-M, Chien L-H, et al. Collateral benefit of COVID-19 control measures on influenza activity, Taiwan. Emerg Infect Dis 2020;26:1928-30.

12 Olsen SJ, Azziz-Baumgartner E, Budd AP, et al. Decreased Influenza Activity During the COVID-19 Pandemic - United States, Australia, Chile, and South Africa, 2020. MMWR Morb Mortal Wkly Rep 2020;69:1305-9.

13 Huang QS, Wood T, Jelley L, et al. Impact of the COVID-19 nonpharmaceutical interventions on influenza and other respiratory viral infections in New Zealand. Nat Commun 2021;12:1-7.

14 Cuadrado-Payán E, Montagud-Marrahi E, Torres-Elorza M, et al. SARS-CoV-2 and influenza virus co-infection. Lancet 2020;395:e84.

15 Antony SJ, Almaghlouth NK, Heydemann EL. Are coinfections with COVID-19 and influenza low or underreported? An observational study examining current published literature including three new unpublished cases. J Med Virol 2020;92:2489-97.

16 Zheng $\mathrm{X}$, Wang $\mathrm{H}$, Su Z, et al. Co-infection of SARS-CoV-2 and influenza virus in early stage of the COVID-19 epidemic in Wuhan, China. J Infect 2020;81:e128-9.

17 Yue $\mathrm{H}$, Zhang $\mathrm{M}$, Xing $\mathrm{L}$, et al. The epidemiology and clinical characteristics of co-infection of SARS-CoV-2 and influenza viruses in patients during COVID-19 outbreak. J Med Virol 2020;92:2870-3

18 Nicholson KG, Wood JM, Zambon M. Influenza. Lancet 2003;362:1733-45.

19 Nair M, Prabhakaran D. Why do South Asians have high risk for CAD? Glob Heart 2012;7:307-14.

20 Murray CJL, Lopez AD, Chin B, et al. Estimation of potential global pandemic influenza mortality on the basis of vital registry data from the 1918-20 pandemic: a quantitative analysis. The Lancet 2006;368:2211-8.

21 Ahmed M, Aleem MA, Roguski K. Estimates of seasonal influenza-associated mortality in Bangladesh, 2010-2012. Influenza and Other Respiratory Viruses, 2017.

22 World Health Organization. COVID-19 Bangladesh situation reports. World Health Organization, 2020. https://www.who.int/docs/ default-source/searo/bangladesh/covid-19-who-bangladeshsituation-reports/who covid-19-update 41_20201207.pdf?sfvrsn= d21baace_7

23 World Health Organization. GISRS sentinel surveillance for COVID-19: frequently asked questions (FAQ). World Health Organization, 2020.

24 Azziz-Baumgartner E, Alamgir ASM, Rahman M, et al. Incidence of influenza-like illness and severe acute respiratory infection during three influenza seasons in Bangladesh, 2008-2010. Bull World Health Organ 2012:90:12-19.
25 Homaira N, Luby SP, Alamgir ASM, et al. Influenza-associated mortality in 2009 in four sentinel sites in Bangladesh. Bull World Health Organ 2012;90:272-8.

26 Ahmed M, Aleem MA, Roguski K, et al. Estimates of seasonal influenza-associated mortality in Bangladesh, 2010-2012. Influenza Other Respir Viruses 2018;12:65-71.

27 World Health Organization. Global epidemiological surveillance standards for influenza. Edited by Influenza WHOWGESSf Geneva, Switzerland: World Health Organization, 2013.

28 Kim D, Quinn J, Pinsky B, et al. Rates of co-infection between SARSCoV-2 and other respiratory pathogens. JAMA 2020;323:2085-6.

29 Khodamoradi Z, Moghadami M, Lotfi M. Co-infection of coronavirus disease 2019 and influenza: a report from Iran. Arch Iran Med 2020;23:239-43.

30 Ma S, Lai X, Chen Z, et al. Clinical characteristics of critically ill patients co-infected with SARS-CoV-2 and the influenza virus in Wuhan, China. Int J Infect Dis 2020;96:683-7.

31 World Health Organization. Coronavirus disease (COVID-2019) Bangladesh situation report \# 4, 2020. Available: https://www. who.int/docs/default-source/searo/bangladesh/covid-19-whobangladesh-situation-reports/who-ban-covid-19-sitrep-04.pdf? sfvrsn=69b6d931_8

32 Akhtar Z, Chowdhury F, Aleem MA, et al. Undiagnosed SARS-CoV-2 infection and outcome in patients with acute MI and no COVID-19 symptoms. Open Heart 2021;8:e001617.

33 Palache A, Oriol-Mathieu V, Fino M, et al. Seasonal influenza vaccine dose distribution in 195 countries (2004-2013): little progress in estimated global vaccination coverage. Vaccine 2015;33:5598-605

34 Hirve S, Lambach P, Paget J, et al. Seasonal influenza vaccine policy, use and effectiveness in the tropics and subtropics - a systematic literature review. Influenza Other Respir Viruses 2016;10:254-67.

35 Yang W, Cao Q, Qin L, et al. Clinical characteristics and imaging manifestations of the 2019 novel coronavirus disease (COVID19):A multi-center study in Wenzhou city, Zhejiang, China. J Infect 2020;80:388-93.

36 Rodriguez-Morales AJ, Cardona-Ospina JA, Gutiérrez-Ocampo $\mathrm{E}$, et al. Clinical, laboratory and imaging features of COVID-19: a systematic review and meta-analysis. Travel Med Infect Dis 2020;34:101623.

37 Li X, Xu S, Yu M, et al. Risk factors for severity and mortality in adult COVID-19 inpatients in Wuhan. J Allergy Clin Immunol 2020;146:110-8.

38 Livingston E, Bucher K. Coronavirus disease 2019 (COVID-19) in Italy. JAMA 2020;323:1335.

39 Huang $\mathrm{C}$, Wang $\mathrm{Y}, \mathrm{Li}$ X, et al. Clinical features of patients infected with 2019 novel coronavirus in Wuhan, China. Lancet 2020;395:497-506

40 Garg S, Kim L, Whitaker M, et al. Hospitalization rates and characteristics of patients hospitalized with laboratory-confirmed coronavirus disease 2019 - COVID-NET, 14 States, March 1-30, 2020. MMWR Morb Mortal Wkly Rep 2020;69:458-64.

41 Sanyaolu A, Okorie C, Marinkovic A, et al. Comorbidity and its impact on patients with COVID-19. SN Compr Clin Med 2020;2:1069-76.

42 Palaiodimos L, Kokkinidis DG, Li W, et al. Severe obesity, increasing age and male sex are independently associated with worse inhospital outcomes, and higher in-hospital mortality, in a cohort of patients with COVID-19 in the Bronx, New York. Metabolism 2020;108:154262.

43 Mead PS, Slutsker L, Dietz V, et al. Food-related illness and death in the United States. Emerg Infect Dis 1999;5:607-25. 